\title{
EVALUATION OF NON-LINEAR PHASE SPACE DISTORTIONS VIA FREQUENCY ANALYSIS
}

\author{
R. Bartolini and F. Schmidt
}

\begin{abstract}
Several methods have been developed recently to measure the frequencies of a time series to much higher precision than ordinary FFT. The decomposition of tracking or experimental data into a Fourier transform can therefore also be done with largely enhanced precision. On the one hand this allows to free these data from complicated phase space distortions. On the other hand, these high precision spectrum lines can be interpreted as being the result of the excitation of certain resonances. In this report we study how this information can be used to tackle resonances. It has to be stressed that this techniques requires no knowledge concerning the simulation model or the accelerator being studied. The only input needed is a series of tracking data or, in the case of the experiment, a set of turn-by-turn data after kicking the beam.
\end{abstract}

Paper presented at the workshop on: "Nonlinear and Collective Phenomena in Beam Physics", Arcidosso, September 1996.

Administrative Secretariat

LHC Division

CERN

CH-1211 Geneva 23

Switzerland

Geneva, 5 February 1997 
A very precise determination of the fundamental frequencies of quasi-integrable systems has been introduced recently and applied to the analysis of the betatron motion in an accelerator $[1,2]$. The aim of this report is to use the improved knowledge about these frequencies for a better understanding of the nonlinear contents of single particle motion in accelerators. An earlier attempt to relate the frequency spectrum to the driving term of a resonance was proposed $[3,4]$ in the framework of the first order perturbation theory with applications to the real spectrum from tracking or experimental turn-by-turn data.

In Sec. 2 we will give a short overview of the theory as introduced by Bengtsson [3]. In Sec. 3.1 we discuss the links between the lines and the resonances as expected from perturbation theory using the Hénon 2D map. In Sec. 3.2 we will show an application for the SPS and for a realistic LHC lattice. For the latter [Sec. 3.3] a correction strategy based on the analysis of the spectral lines allows to correct third-order resonances. The implications concerning the dynamic aperture are discussed.

\section{Spectral analysis via first order perturbation theory}

Close to a single resonance, the quasi-integrable system can be represented in first order perturbation theory, in the following form:

$$
H\left(J_{x}, J_{z}, \phi_{x}, \phi_{z} ; s\right)=\nu_{x} J_{x}+\nu_{z} J_{z}+D_{T}\left(J_{x}, J_{z}\right) \cos \psi
$$

where $\left(\nu_{x}, \nu_{z}, J_{x}, J_{z}, \phi_{x}, \phi_{z}\right)$ are the tunes and the action-angle variables of the unperturbed system. The phase term $\psi$ is

$$
\psi=n_{x} \phi_{x}+n_{z} \phi_{z}+\phi_{k}-p \frac{s-s_{0}}{R}
$$

with $s, \phi_{k}$ and $R$ the longitudinal position, initial phase and averaged radius respectively. $D_{T}$ is the amplitude dependent driving term and the resonance condition of the frequencies is:

$$
n_{x} \nu_{x}+n_{z} \nu_{z}+p=0 \quad n_{x}, n_{z}, p \in \mathbf{Z}
$$

where $n_{x}+n_{z}=N$ is the order of the resonance. J. Bengtsson [3], showed that the motion generated by Eq. 1 gives rise to a Fourier spectrum, each of its components being related to a particular resonance. The following table shows which lines are expected in first order for the horizontal and vertical plane respectively:

\begin{tabular}{||c|c||}
\hline Horizontal & Vertical \\
\hline$\left(n_{x} \pm 1\right)\left(\nu_{x}+\Delta \nu_{x}\right)+n_{z}\left(\nu_{z}+\Delta \nu_{z}\right)$ & $n_{x}\left(\nu_{x}+\Delta \nu_{x}\right)+\left(n_{z} \pm 1\right)\left(\nu_{z}+\Delta \nu_{z}\right)$ \\
\hline
\end{tabular}

where the $\Delta \nu_{x}$ and $\Delta \nu_{z}$ are the amplitude dependent detuning terms. Notice that in the line spectrum the resonance conditions are found for the exact frequencies while the unperturbed tunes are used when applying the perturbation theory.

The actual procedure to obtain the full spectrum of the betatron motion goes as follows [1]: the time series of tracking (or experimental) data is analysed with the algorithms for the precise measure of the betatron tune. They provide the main frequency (i.e. the tune) and the corresponding Fourier coefficient. By subtracting from the time series this tune line, using the proper amplitude and phase, we obtain a new signal of 
equal length which can be reanalysed in the same way. This iterative procedure provides the set of frequencies contained in the betatron motion.

It is easily verified that the frequencies can be expressed as linear combinations of the fundamental tunes $\nu_{x}$ and $\nu_{y}$ and the decomposition of the signal reads:

$$
x(n)=\sum_{j=1}^{M} a_{j} e^{i\left[2 \pi\left(m_{j} \nu_{x}+n_{j} \nu_{z}\right) n+\phi_{j}\right]} \quad m_{j}, n_{j} \in \mathbf{Z}
$$

where the $a_{j}$ and $\phi_{j}$ are amplitude and phase of the corresponding spectral line. It should be mentioned here that the algorithms used for the high frequency analysis rely on the hypothesis that the time series is a quasi-periodic signal of length $T$ with frequencies separated by a distance larger than $1 / T$. This implies that, in general, regular motion can always be treated by choosing large enough sample lengths. However, in the chaotic regime the method will break down since the above condition is not fulfilled.

\section{Application to models}

The identification of the spectral lines in terms of resonances is only partially solved by perturbation theory owing to the complexity of the problem. For the 2D Hénon map we have systematically studied amplitude and phase of various lines and their relation to first order perturbation theory. The beneficial effects of reducing phase space distortion by subtraction of spectral lines are demonstrated using more realistic models of the SPS and the LHC. Finally we discuss how the lines are used to correct resonances of the LHC lattice.

\subsection{The Hénon 2D map}

The Hénon map can be represented by a sextupolar kick followed by a rotation:

$$
\left\{\begin{array}{l}
x^{\prime}=\cos \left(2 \pi \nu_{x}\right) \cdot x+\sin \left(2 \pi \nu_{x}\right) \cdot\left(p_{x}+x^{2}\right) \\
p_{x}^{\prime}=-\sin \left(2 \pi \nu_{x}\right) \cdot x+\cos \left(2 \pi \nu_{x}\right) \cdot\left(p_{x}+x^{2}\right)
\end{array}\right.
$$

Tracking data were generated with different starting amplitudes and different values of the tune and were analysed with the procedure described in Sec. 2. According to the first order perturbation theory only the resonances of order 3, and the sub-resonances of order 1 can be excited. Using the results of Sec. 2 we expect to find the following lines:

\begin{tabular}{||c|c||}
\hline 3-rd order & 1-st order \\
\hline $\pm 2 \nu_{x}$ & 0 \\
$\pm 4 \nu_{x}$ & $\pm 2 \nu_{x}$ \\
\hline
\end{tabular}

The results are summarised in Fig. 1. It shows the dynamic aperture of the model and the behaviour of the phase of the different spectral lines with respect to the tune variation. Characteristic jumps in the phase of the lines by 180 degrees are found at tune values for which Bengtsson has predicted resonances. Actually many more lines are found due to terms that have been neglected by the first order perturbation theory. For example the lines $\pm 3 \nu_{x}$ may be explained by a naive application of Bengtsson derivation to the single resonance Hamiltonian arising from the model in second order.

In general it is difficult to establish a one-to-one correspondence between lines and resonances, because the existence of a certain resonant term in the Hamiltonian may contribute to different lines. In particular this seems to be true for higher order resonances. 


\begin{tabular}{|c|c|c|}
\hline Stability Border & Uncorrected LHC lattice & $\begin{array}{c}\text { Correction } \\
\text { of }(3,0) \&(1,2) \\
\text { Resonance }\end{array}$ \\
\hline \hline Regular Motion & 15.5 & 16.9 \\
\hline Strong Chaos & 16.0 & 17.1 \\
\hline Lost before 1000 Turns & 16.9 & 18.0 \\
\hline
\end{tabular}

Table 1: Improvement of Dynamic Aperture due to Resonance Correction

\subsection{Reduction of Phase Space Deformations}

In a first example [LHC case in Fig. 2a, c] the doughnut shaped horizontal phase space is reduced to a near perfect circle by removing the first 100 dominant lines. It goes without saying that the tune line has to be kept. This procedure does not introduce high order distortions which tend to spoil the usefulness of perturbative techniques like Normal Form.

The strong reduction of phase space distortion can be applied to sharpen the method for detecting the onset of chaos [6]. In Fig. $2 \mathrm{~b}$ a typical case is shown of the evolution of the angular distance in phase space of initially close-by particles. In the case of regular motion a linear increase of this distance is expected. The large variations of the distance may make it difficult however to decide about the nature of the particle motion. The subtraction of lines (compare [part d.)] to [part b.)]) offers an easy and reliable method to reduce these variations.

The most difficult test is the study of motion close to resonance structures. The large five islands [SPS case in Fig. 2a] can indeed be reduced to points by the subtraction of lines as seen in part b.). The one essential precondition of this method is however the existence of a well defined tune. The method therefore fails in the case of chaotic motion, here achieved by approaching the vicinity of the separatrix. The subtraction of 100 lines that transform [part c.)] into [part d.)] does no longer simplify the complexity in phase space.

\subsection{The correction of the resonances in the LHC}

A possible exploitation of the techniques discussed so far consist in the correction of the resonance contributions generated by the nonlinear elements in an accelerator lattice. For this purpose we analysed tracking data of a realistic LHC model.

Following well established strategies for the correction of the resonances [5] we tried to identify the location and the strength of a set of corrector families to compensate the third order resonances $(3,0)$ and $(1,2)$. A family of sextupolar spool pieces, normally used to correct the average $b_{3}$ component along the lattice was split into several families to compensate the cosine and sine term of the two resonances. Using tracking data at each location of the correctors we were able to identify the best places for correctors, i.e. longitudinal locations where the oscillations of the lines have their extreme values [Fig. 3]. Two resonances were corrected simultaneously each with two correction families while keeping the $b_{3}$ corrected on average. We managed to reduce by more that $50 \%$ the amplitude of the lines. The resulting reduction of the phase space distortions is clearly visible in Fig. (4). In the tracking [Tab. 1] we could demonstrate that the double resonance correction led to an improvement of the dynamic aperture of almost $10 \%$. 


\section{Conclusions}

We have shown that the tune line spectrum can serve as a powerful tool to deal with strong nonlinearities in single particle motion. It is appealing for the accelerator designer to have a tool that works without involved mathematical apparatus. It works very well in simulations and is expected to be equally useful in machine experiments. We have demonstrated that these lines can be used to suppress unwanted phase space distortions and to correct resonances in a non-perturbative manner.

\section{References}

[1] J. Laskar, Physica D 67, 257-281 (1993).

[2] R. Bartolini, A. Bazzani, M. Giovannozzi, W. Scandale and E. Todesco, Part. Acc. 56, 167-199 (1996).

[3] J. Bengtsson, CERN 88-05 (1988).

[4] A.Ando, Part. Acc. 15, 177-207 (1984).

[5] G. Guignard, CERN 76-12 (1976).

[6] F. Schmidt, F. Willeke and F. Zimmermann, Part. Accel. 35, 249-256 (1991). 

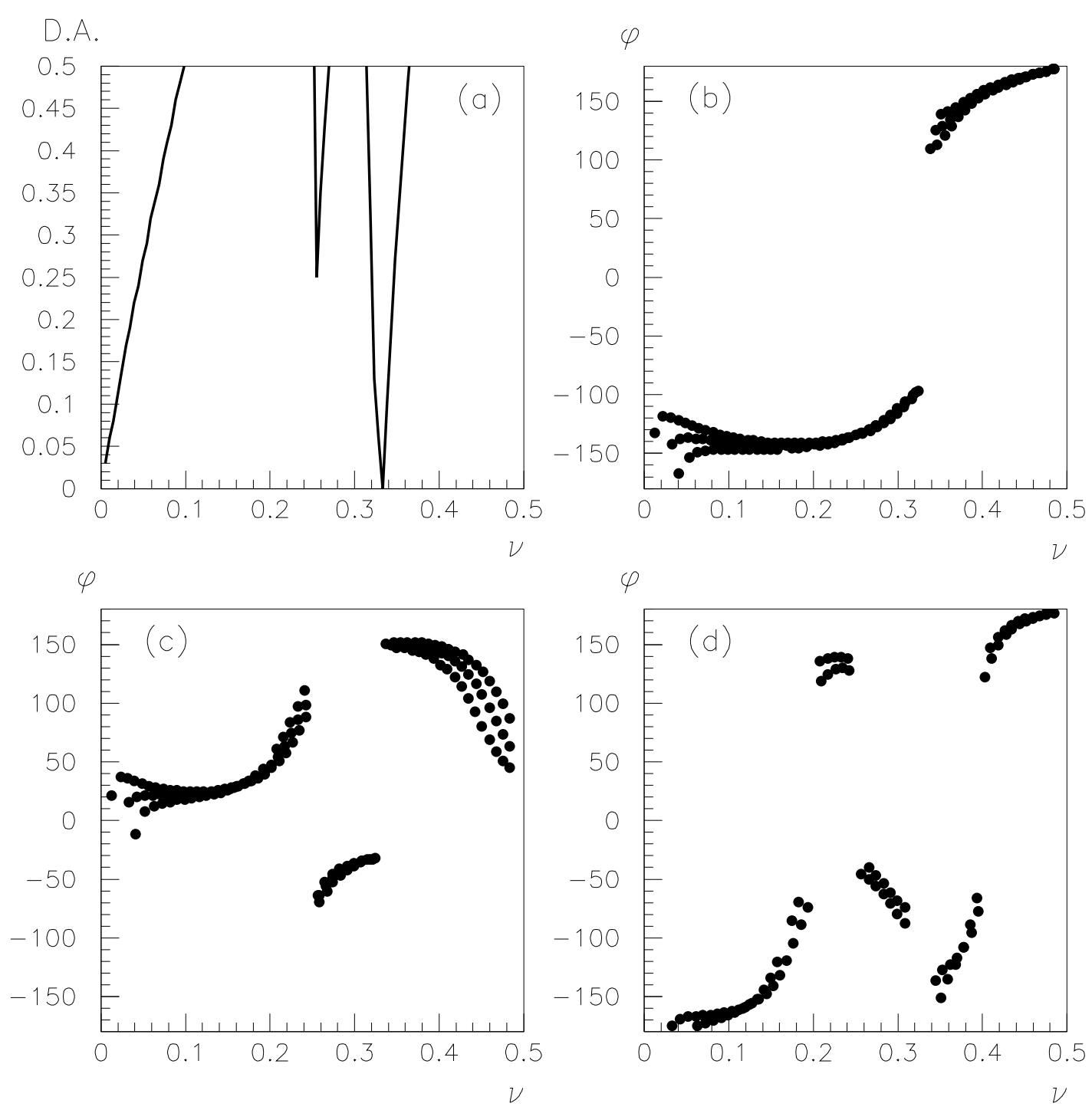

Figure 1: The Hénon 2D map

Part a.): Dynamic Aperture versus Tune

Part b.): Phase dependence of the line $\pm 2 \nu$

Part c.): same for $\pm 3 \nu$

Part d.): same for $\pm 4 \nu$ 
a.)

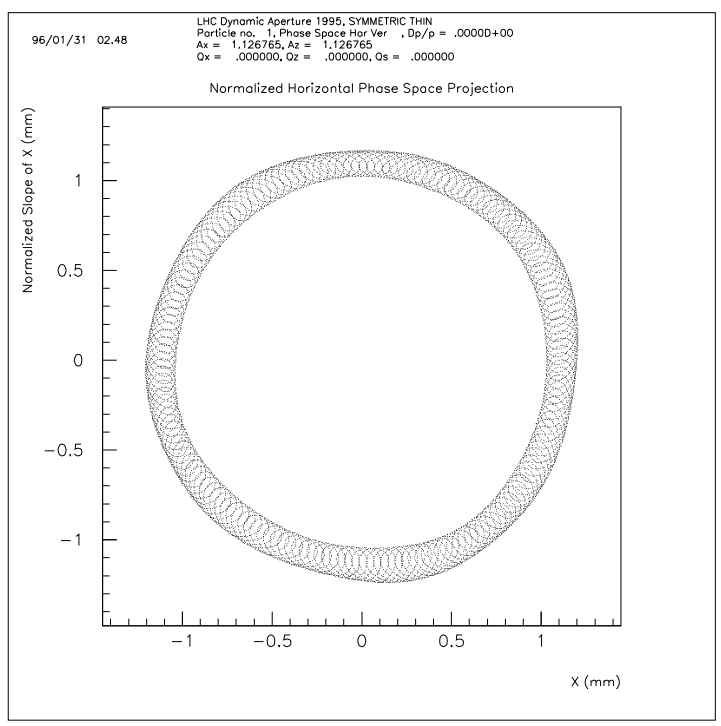

c.)

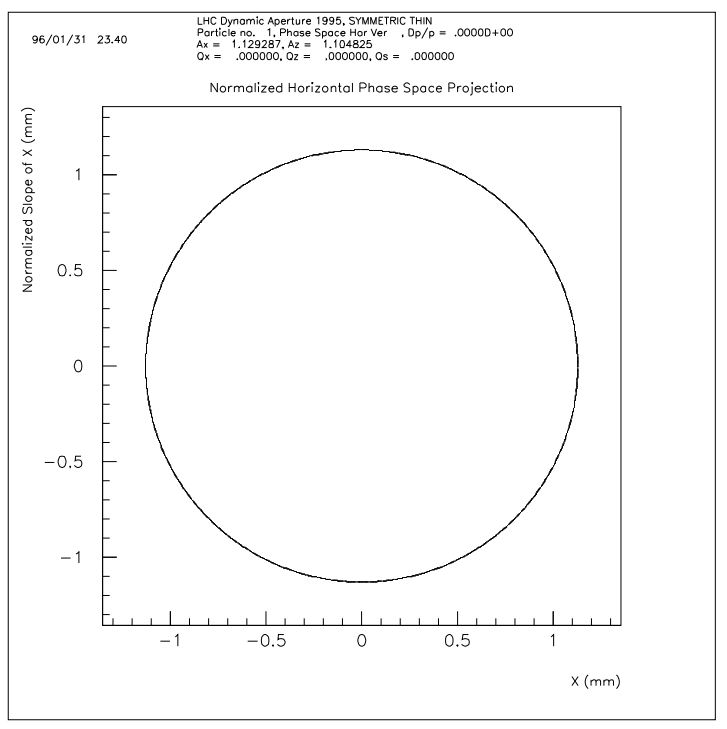

b.)

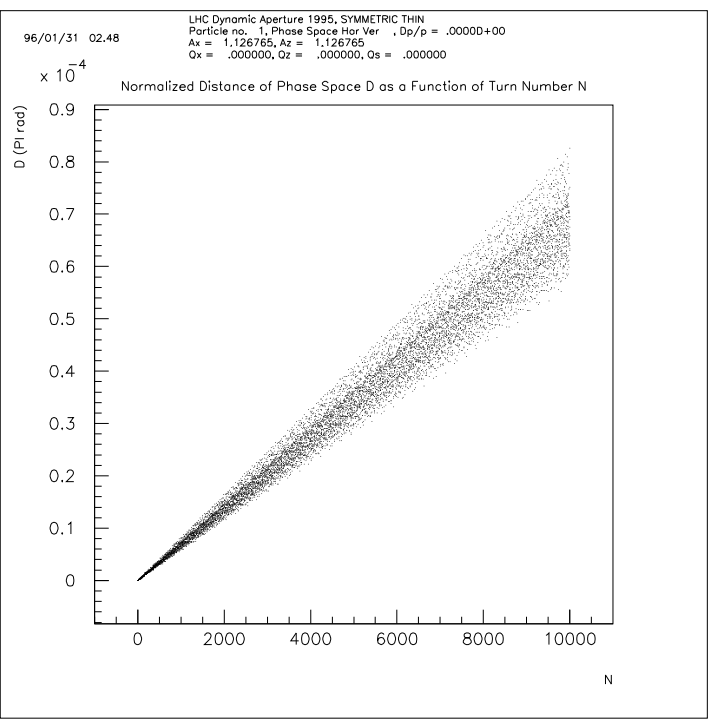

d.)

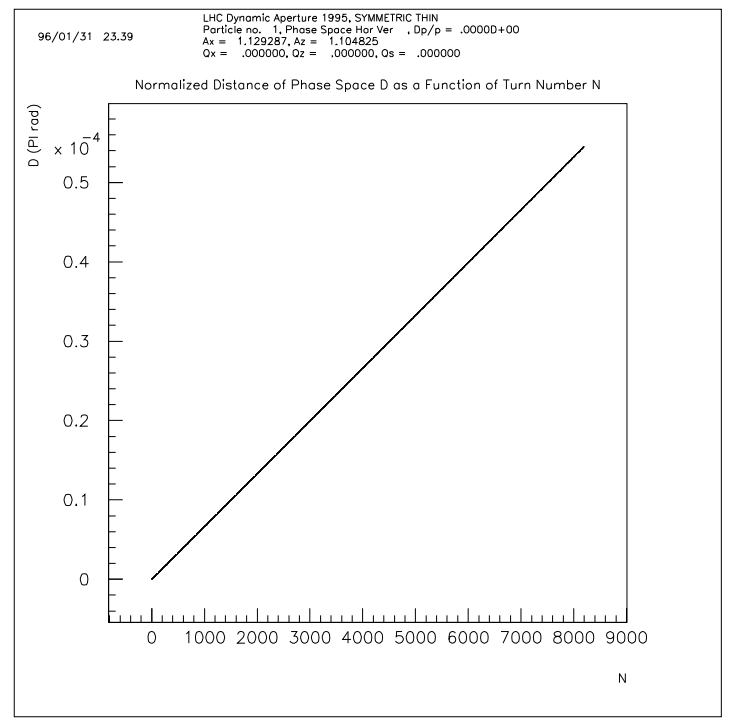

Figure 2: Reducing Phase Space Distortions by Subtraction of dominant Lines

Part a.) shows a typical horizontal phase space plot of nonlinear particle motion in a LHC structure. The linear increase of the distance of two initially close-by particles indicates that the motion is regular, that is to say stable forever. Taking out the most dominant lines (with the exception of the tune line) reduces the phase space to a near perfect circle [part c.)]. Moreover the increase of the distance in phase space, which is shaped like a wedge as seen in part b.), reduces to a thin line after the subtraction [part d.)]. 
a.)

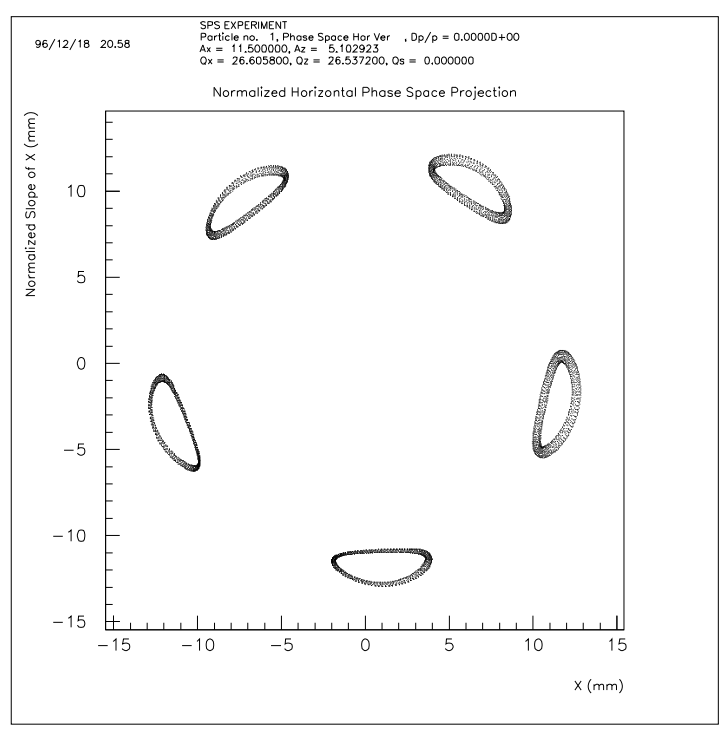

c.)

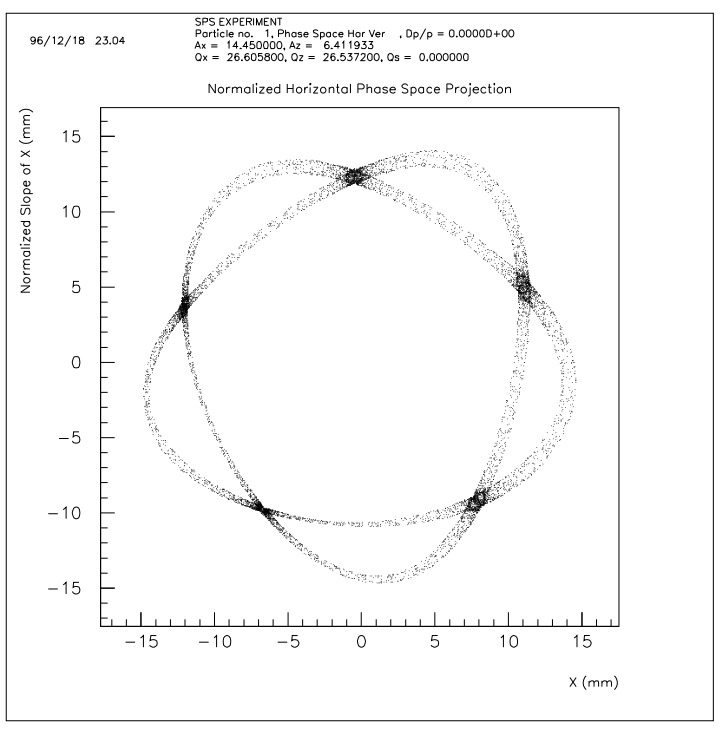

b.)

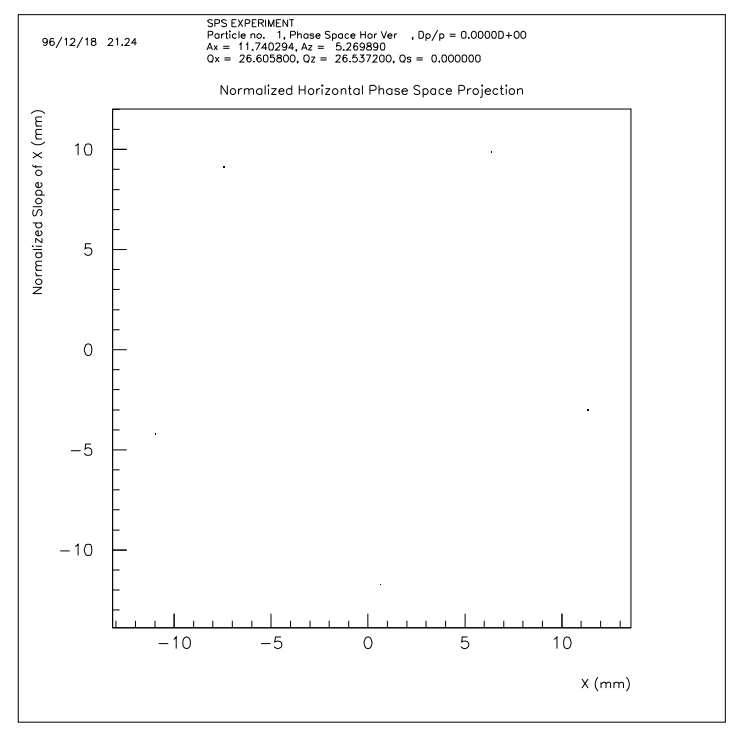

d.)

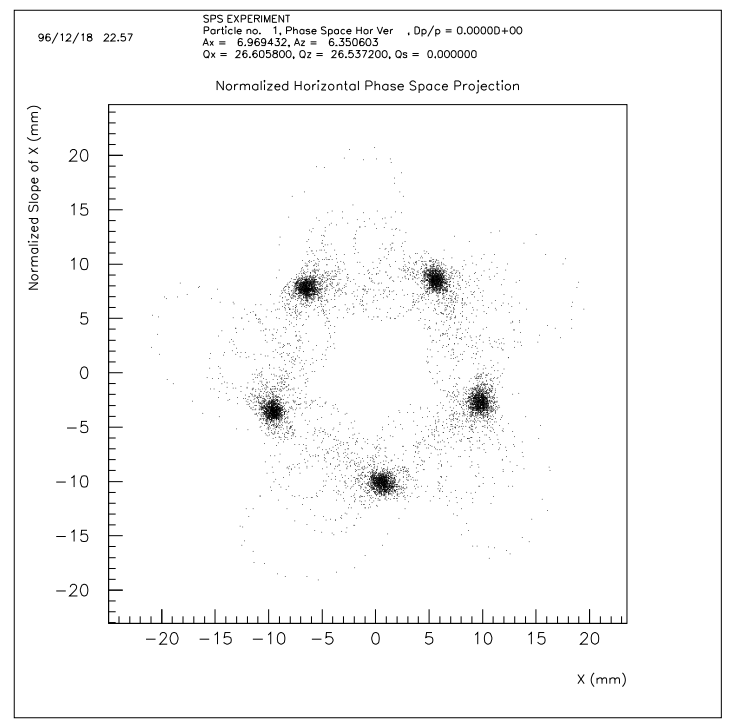

Figure 3: Reducing Phase Space Distortions close to $5^{\text {th }}$ Order Resonance The motion close to a $5^{\text {th }}$ order resonance is shown in part a.). Taking out the 100 largest lines while keeping the tune line reduces the islands to points which are just visible in part b.). The method works of course only for regular motion. Once chaotic motion is considered, here by approaching the separatrix [part c.)], the subtraction of lines no longer leads to point-like objects [part d.)]. On the contrary, one can argue that the phase space has become more distorted after this subtraction. 


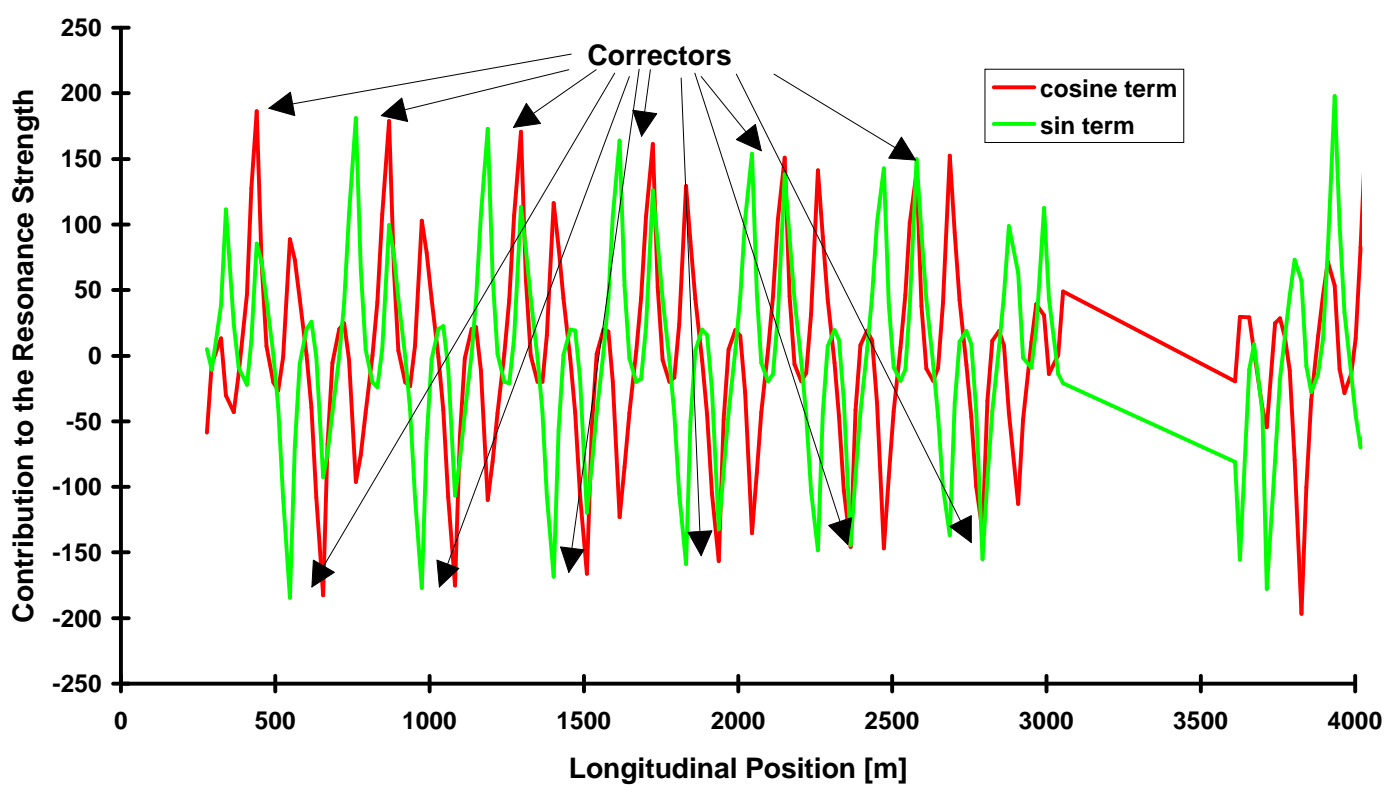

Figure 4: Choosing best places for correcting the $(3,0)$ resonances with sextupoles

a.)

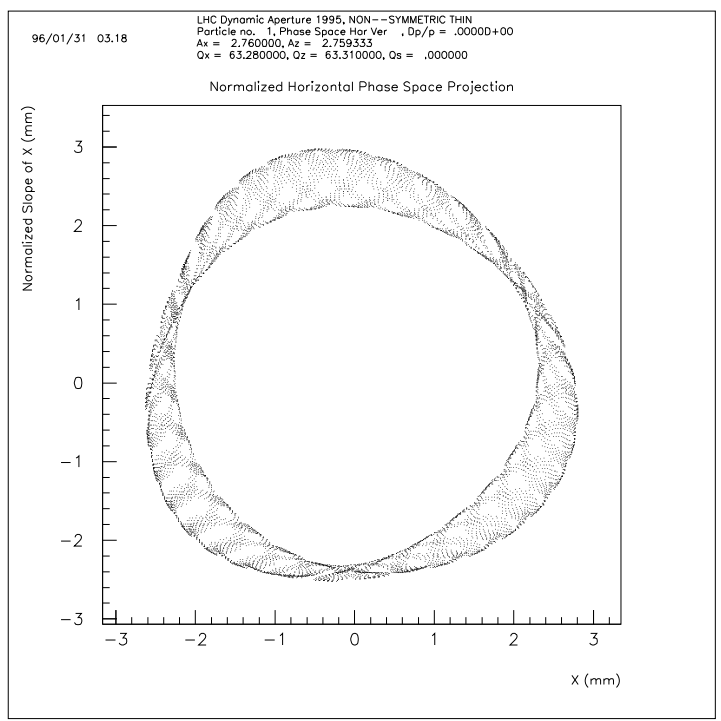

b.)

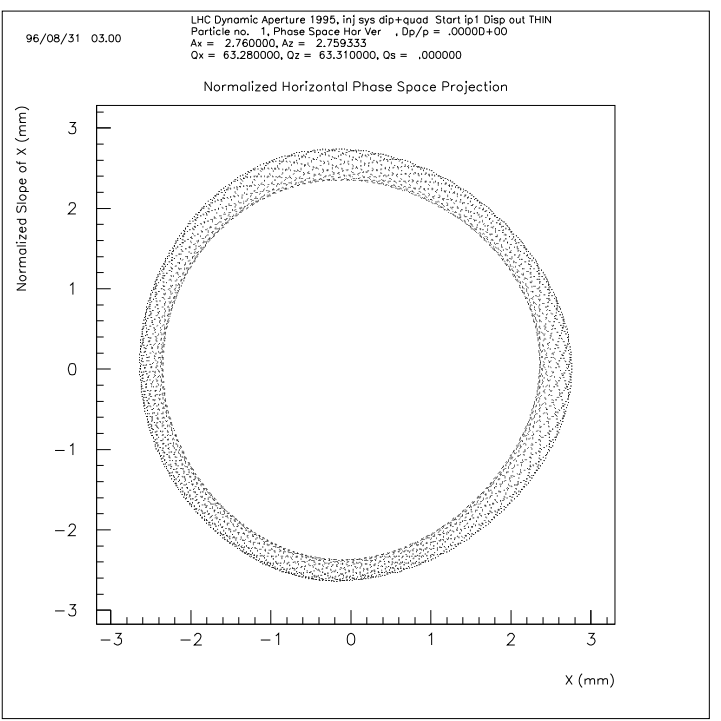

Figure 5: Reduction of Phase Space Distortion due to Correction of Resonances In part a.) the horizontal phase space of particle motion is shown in a LHC lattice with the $(3,0)$ and the $(1,2)$ resonance strongly excited. These resonances have been corrected resulting in the corresponding phase space projection as depicted in part b.). 